\title{
ON PROPER HOLOMORPHIC MAPPINGS FROM DOMAINS WITH T-ACTION
}

\author{
BERNARD COUPET, YIFEI PAN AND ALEXANDRE SUKHOV
}

\begin{abstract}
We describe the branch locus of a proper holomorphic mapping between two smoothly bounded pseudoconvex domains of finite type in $\mathbb{C}^{2}$ under the assumption that the first domain admits a transversal holomorphic action of the unit circle. As an application we show that any proper holomorphic self-mapping of a smoothly bounded pseudoconvex complete circular domain of finite type in $\mathbb{C}^{2}$ is biholomorphic.
\end{abstract}

\section{$\S 1$. Introduction}

In the present paper we study proper holomorphic mappings between smoothly bounded pseudoconvex domains of finite type in $\mathbb{C}^{2}$. We begin with the description of the branch locus of a proper holomorphic mapping. Let $\Omega$ be a smoothly bounded pseudoconvex domain of finite type in $\mathbb{C}^{2}$. It follows by $[3,7]$ that the automorphism group action $\operatorname{Aut}(\Omega) \times \Omega \longrightarrow \Omega$, $(f, z) \mapsto f(z)$ extends smoothly to $\bar{\Omega}$. Thus we can assume that $\operatorname{Aut}(\Omega)$ acts smoothly on $\bar{\Omega}$ and in particular on $\partial \Omega$. We say (see $[3,4,27]$ ) that a subgroup $G$ of $\operatorname{Aut}(\Omega)$ acts transversally on $\partial \Omega$ if for every point $p \in \partial \Omega$ the image of the tangent mapping $\left(\Psi_{p}\right)^{*}: T_{e} G \longrightarrow T_{p}(\partial \Omega)$ associated to the mapping $\Psi_{p}: G \longrightarrow \partial \Omega, f \mapsto f(p)$ is not contained in the holomorphic tangent space $H_{p}(\partial \Omega)$. We will denote by $\mathbf{T}$ the Lie group of the unite circle. If $\mathbf{T}$ is a subgroup of $\operatorname{Aut}(\Omega)$ and acts transversally on $\partial \Omega$, we will simply say that $\Omega$ admits a transversal $\mathbf{T}$-action.

Let $f: \Omega \longrightarrow D$ be a proper holomorphic mapping between two domains $\Omega$ and $D$. We will denote by $J_{f}(z)$ the Jacobian determinant of $f$ and by $V_{f}=\left\{z \in \Omega: J_{f}(z)=0\right\}$ the branch locus of $f$.

Our first main result is the following

THEOREM 1.1. Let $f: \Omega \longrightarrow D$ be a proper holomorphic mapping between two smoothly bounded pseudoconvex domains of finite type in $\mathbb{C}^{2}$.

Received February 18, 1998. 
Suppose that $\Omega$ admits a transversal $\mathbf{T}$-action. Then for any irreducible component $V$ of the branch locus $V_{f}$ the following holds:

(i) $(V, \partial V)$ is a smooth manifold with boundary in a neighborhood of every point in $\bar{V} \cap \partial \Omega$.

(ii) $\partial V:=\bar{V} \backslash V$ is a finite disjoint union of $\mathbf{T}$-orbits.

It is well-known [1] that any proper holomorphic self-mapping of the unit ball is biholomorphic. This important result was extended to larger classes of domains by several authors $[6,8,10,13,22,26]$. One of the natural conjectures here is to show that any proper holomorphic self-mapping of a pseudoconvex domain with smooth finite type boundary is biholomorphic (this question still remains open even in dimension 2). In our paper we establish a result confirming this general conjecture.

THEOREM 1.2. Let $\Omega$ be a smoothly bounded pseudoconvex complete circular domain of finite type in $\mathbb{C}^{2}$. Then every proper holomorphic selfmapping of $\Omega$ is a biholomorphism.

In contrast with the $\mathbf{T}^{2}$ action case a regularity of the boundary is essential here. Indeed, a basin of attraction of a polynomial homogeneous complex dynamic system in $\mathbb{C}^{2}$ is a complete circular domain [23]; this gives a large class of examples of circular domains with proper holomorphic self-mappings which are not automorphisms. For instance, there exists a complete circular domain $D$ in $\mathbb{C}^{2}$ with real analytic strictly pseudoconvex boundary outside of the union of three circles (where the boundary is not smooth) such that there is a proper holomorphic self-mapping of $D$ which is not biholomorphic [14] .

The basic idea in proof of Theorem 1.1 given in Section 2 and 3 is a special version of the scaling method developed in $[17,18]$. One can consider this method as a quantitative version of deformation of a complex structure which reduces the determination of the branch locus to a very special class of domains with algebraic boundaries. Theorem 1.2 then follows by elementary complex dynamics arguments in Section 4.

\section{$\S 2$. Branching of holomorphic mappings between algebraic domains}

This section is devoted to the study of holomorphic mappings between algebraic domains in $\mathbb{C}^{2}$. The general situation will be reduced to this case in the next section. 
We recall certain general facts about boundary behavior of proper holomorphic mappings. Let $f: D_{1} \longrightarrow D_{2}$ be a proper holomorphic mapping between two pseudoconvex smoothly bounded domains in $\mathbb{C}^{2}$. We suppose that $f$ is smooth up to the boundary. Let $r_{j}$ be the defining function of $D_{j}$. Following $[6,8]$, we consider the Levi-determinant of $D_{j}$ defined as follows:

$$
\Lambda_{\partial D_{j}}(p)=-\operatorname{det}\left(\begin{array}{ccc}
0 & \frac{\partial r_{j}}{\partial z} & \frac{\partial r_{j}}{\partial w} \\
\frac{\partial r_{j}}{\partial \bar{z}} & \frac{\partial^{2} r_{j}}{\partial z \partial \bar{z}} & \frac{\partial^{2} r_{j}}{\partial z \partial \bar{w}} \\
\frac{\partial r_{j}}{\partial \bar{w}} & \frac{\partial^{2} r_{j}}{\partial w \partial \bar{z}} & \frac{\partial^{2} r_{j}}{\partial w \partial \bar{w}}
\end{array}\right)
$$

Obviously $\Lambda_{\partial D_{2}}(f(p))\left|J_{f}(z)\right|^{2}=\Lambda_{\partial D_{1}}(p)$ for any $p \in \partial D_{1}$.

For any boundary point $p \in \partial D_{j}$ we consider also the order of vanishing of $\Lambda_{D_{j}}$ at $p$ denoted by $\tau_{\partial D_{j}}(p)$, which is defined as follows: we choose smooth real coordinates $x=\left(x_{1}, x_{2}, x_{3}\right)$ on $\partial D_{j}$ such that $p$ corresponds to $x=0$, and the formal power series $\Lambda_{D_{j}}(x)=\sum_{j=0}^{\infty} \sum_{|\alpha|=j} a_{\alpha} x^{\alpha}$, where $\alpha=\left(\alpha_{1}, \alpha_{2}, \alpha_{3}\right)$ is a multi-index and $|\alpha|=\alpha_{1}+\alpha_{2}+\alpha_{3}$. We set $\tau_{\partial D_{j}}(p)=$ $\min \left\{|\alpha|: a_{\alpha} \neq 0\right\}$ (of course, this definition does not depend on the choice of coordinates). The following properties of $\tau$ are well known (see $[6,10])$ :

(1) $\tau_{\partial D_{j}}(p)$ is an upper-semicontinuous function on $\partial D_{j}$.

(2) $\tau_{\partial D_{2}}(f(p)) \leq \tau_{\partial D_{1}}(p)$ and the equality holds if and only if $\overline{V_{f}}$ does not contain $p$ i.e. $f$ is a diffeomorphism on the boundary near $p$.

The main purpose of this section is to study the structure of the branch locus of a proper holomorphic mapping $f$ between rigid algebraic domains $\Omega=\left\{(z, w) \in \mathbb{C}^{2}: \rho(z, w)=\operatorname{Im} w+P(z)<0\right\}$ and $D=\{(z, w) \in$ $\left.\mathbb{C}^{2}: \phi(z, w)=\operatorname{Im} w+Q(z)<0\right\}$, where $P, Q$ are non identically zero subharmonic polynomials without purely harmonic terms.

The set of weakly pseudoconvex points of $\partial \Omega$ will be denoted by $w(\partial \Omega)$. One has $w(\partial \Omega)=\{z \in \mathbb{C}:(\Delta P)(z)=0\} \times \mathbb{R}$.

Let us consider the set $\Sigma_{\Omega} \in \mathbb{C}$ of singular points of the set $S=\{z \in$ $\mathbb{C}:(\Delta P)(z)=0\}$, i.e. the set of points in $\mathbb{C}$ such that $S$ is not a smooth curve in any neighborhood of such a point. Note that $\Sigma_{\Omega}$ is finite (as an algebraic set of dimension 0 ).

Proposition 2.1. Let $U$ be a neighborhood of the origin and $f: \Omega \cap$ $U \longrightarrow D$ be a holomorphic mapping verifying the following property: for every point $p \in U \cap \partial \Omega$ and any sequence of points $\left(p^{j}\right)_{j}$ in $\Omega$ converging 
to $p$ the sequence of images $\left(f\left(p^{j}\right)\right)_{j}$ has no cluster points in D. Then for every open $U^{\prime} \subset U$ the branch locus $U^{\prime} \cap V_{f}$ in $U^{\prime}$ is contained in the union of complex lines $\cup_{z_{j} \in \Sigma_{\Omega}}\left\{\left(z_{j}, w\right) \in \mathbb{C}^{2}: \operatorname{Im} w<-P\left(z_{j}\right)\right\}$.

The following corollary considers the case useful for the proof of our main results:

Corollary 2.2. If $P$ is homogeneous, then $V_{f}$ is contained in the half-plane $\{(0, w): \operatorname{Im} w<0\}$.

\section{Proof of Proposition 2.1.}

We have $S=S_{1} \cup \ldots \cup S_{k} \cup \Sigma_{\Omega}$, where $S_{j}$ are smooth connected real algebraic curves. We note that the order of vanishing of the laplacian $\Delta P$ is constant on every $S_{j}$ by the connectivity.

Hence, we have $w(\partial \Omega)=\cup_{j=1}^{N}\left(S_{j} \times \mathbb{R}\right) \cup\left(\Sigma_{\Omega} \times \mathbb{R}\right)$ and $\tau(p)$ is constant on every totally real manifold $S_{j} \times \mathbb{R}$.

Let us recall also the following property of the mapping $f$ established in Proposition 2.2 and Lemma 4.1 of [17]: if $p$ is a boundary point of $\Omega$ and there exists a sequence of points $\left(p^{j}\right)_{j}$ in $\Omega$ converging to $p$ such that the sequence of images $\left(f\left(p^{j}\right)\right)_{j}$ converges to a finite boundary point of $\partial D$, then $f$ extends continuously up to the boundary in a neighborhood of $p$; then it follows by Lemma 6.2 of [18] that $f$ extends to $\mathbb{C}^{2}$ as an algebraic mapping i.e. its graph is contained in a complex algebraic 2-dimensional variety $X$ in $\mathbb{C}^{2} \times \mathbb{C}^{2}$. Moreover, it follows by Proposition 6.3 of [18] and [11] that $f$ is smooth up to the boundary in a neighborhood of $p$ and then by Lemma 2.1 of [18] the Jacobian determinant $J_{f}$ of $f$ does not vanishes identically. Thus, $U \cap \partial \Omega$ is a disjoint union of two subsets: the subset $A$ of points where $f$ extends smoothly up to the boundary and the subset $B$ of points $b \in U \cap \partial \Omega$ such that $\lim _{(z, w) \longrightarrow b}|f((z, w))|=\infty$. It was shown in Lemma 4.1 of [17] that $A$ is an (non-empty) open dense subset of $U \cap \partial \Omega$. We will call $B$ the "pull-back of infinity" and will denote by $f^{-1}(\infty)$.

We denote by $\mathbb{C} P^{2}$ the complex 2-dimensional projective space and by $\hat{X}$ the projective closure of $X$ in $\mathbb{C} P^{2} \times \mathbb{C} P^{2}$ which is an irreducible complex 2-dimensional projective variety. Let $\pi_{\Omega}$ (resp. $\left.\pi_{D}\right)$ be the natural projection of $\hat{X}$ to the copy of $\mathbb{C} P^{2}$ containing $\Omega$ (resp. $D$ ) (or more precisely its image under the canonical embedding $\left.i: \mathbb{C}^{2} \hookrightarrow \mathbb{C} P^{2}\right)$. Since $J_{f}$ does not vanishes identically, the composition $\pi_{D} \circ \pi_{\Omega}^{-1}: \mathbb{C} P^{2} \longrightarrow \mathbb{C} P^{2}$ is a proper holomorphic correspondence (see [24]) and in particular, surjective. Then 
$\pi_{\Omega} \circ \pi_{D}^{-1}\left(\mathbb{C} P^{2} \backslash i\left(\mathbb{C}^{2}\right)\right)$ is an complex algebraic curve $\gamma$ in $\mathbb{C} P^{2}$ containing $f^{-1}(\infty)$. Since $\partial \Omega$ is of finite type, the intersection $\gamma^{\prime}=\gamma \cap(U \cap \partial \Omega)$ is at most a real algebraic curve.

LEMMA 2.3. The closure $\overline{V_{f}}$ does not intersect the set of strictly pseudoconvex points in $\partial \Omega$.

Proof. Let $p$ be a strictly pseudoconvex point in $\overline{V_{f}} \cap U \cap \partial \Omega$ and $W$ be its neighborhood which does not intersect $w(\partial \Omega)$. If there exists a point $p^{\prime} \in \overline{V_{f}} \cap W$ which is not in $f^{-1}(\infty)$, then it follows by [17] that $f\left(p^{\prime}\right)$ is a strictly pseudoconvex point in $\partial D$ and $f$ extends to a biholomorphism in a neighborhood of $p^{\prime}$ : a contradiction.

Thus, it suffices to establish the following

Claim. For any open subset $W$ of $\partial \Omega \cap U$ the intersection $\overline{V_{f}} \cap W \cap \partial \Omega$ is not contained in $f^{-1}(\infty)$.

For the proof assume by contradiction that $\overline{V_{f}} \cap W \cap \partial \Omega$ is contained in $f^{-1}(\infty)$. Since $f$ extends to an algebraic mapping, $V_{f}$ is a piece of an complex algebraic subset in $\mathbb{C}^{2}$ and the set of its non-regular points $Y$ is finite. It follows by the maximum principle applied to the restriction $\rho \mid V_{f}$ that the intersection $\overline{V_{f}} \cap \partial \Omega$ cannot contain only point from $Y$. Thus, one can assume that there exists a point $p^{\prime}$ in $\overline{V_{f}} \cap W \cap \partial \Omega$ such that $V_{f}$ extends to a neighborhood of $p^{\prime}$ as a smooth complex manifold $\tilde{V}_{f}$. Now the wellknown argument of [5] using the Hopf lemma shows that (moving slightly $p^{\prime}$ ) one can assume that $\tilde{V}_{f}$ intersects $\partial \Omega$ transversally at $p^{\prime}$.

Let $g(z, w)$ is a holomorphic function on $D,|g(z, w)|<1$ on $D$ and $g(z, w) \longrightarrow 1$ as $|(z, w)| \longrightarrow \infty$ (see [17]). Let us consider the composition $g \circ f$. Since $\overline{V_{f}} \cap \partial \Omega$ is contained in $f^{-1}(\infty),(g \circ f)(z, w) \longrightarrow 1$ as $(z, w)$ tends to $\overline{V_{f}} \cap \partial \Omega$; then it follows by the boundary uniqueness theorem that $(g \circ f) \mid V_{f}$ is equal to 1 identically : a contradiction, and we get the claim.

Thus, we have the inclusion $\overline{V_{f}} \cap U \cap \partial \Omega \subset w(\partial \Omega)$.

Q.E.D.

Lemma 2.4. The intersection $\bar{V}_{f} \cap\left(S_{j} \times \mathbb{R}\right)$ is empty for every $j$.

Proof. Suppose by contradiction that $\overline{V_{f}} \cap\left(S_{j} \times \mathbb{R}\right)$ contains a point $p$ for some $j$. Then $\overline{V_{f}} \cap \partial \Omega$ is contained in $\left(S_{j} \times \mathbb{R}\right)$ near $p$; it follows by the Claim that there exists a point $q$ in $\bar{V} \cap S_{j} \times \mathbb{R}$ such that $f(q)$ is 
finite and $f$ extends smoothly up to the boundary near $q$. Since $J_{f}$ does not vanish identically on $S_{j} \times \mathbb{R}$ by the boundary uniqueness theorem, there exists a sequence $\left(q^{k}\right)$ in $S_{j}$ converging to $q$ such that $J_{f}\left(q^{k}\right) \neq 0$. Therefore, $\tau_{\partial \Omega}\left(q^{k}\right)=\tau_{\partial D}\left(f\left(q^{k}\right)\right)$. On the other hand, $\tau_{\partial D}(f(q))<\tau_{\partial \Omega}(q)$. Since $\tau_{\partial \Omega}$ is constant on $S_{j}$, we have $\tau_{\partial D}(f(q))<\tau_{\partial \Omega}\left(q^{k}\right)=\tau_{\partial D}\left(f\left(q^{k}\right)\right)$. This is a contradiction since the function $\tau_{\partial D}$ is upper semicontinuous.

Q.E.D.

Now the desired proposition follows by the uniqueness theorem.

\section{$\S 3$. Scaling and branching}

We begin with the following local version of Theorem 1.1:

Proposition 3.1. Let $D_{1}=\left\{(z, w) \in W: \operatorname{Im} w+P_{2 m}+\varphi(z)<0\right\}$ be a smooth pseudoconvex finite type domain in a neighborhood $W$ of the origin, $\varphi(z)=o\left(|z|^{2 m}\right), P_{2 m}$ is a non-zero subharmonic polynomial without purely harmonic terms and $D_{2}$ be a smoothly bounded pseudoconvex finite type domain. Let $f: D_{1} \longrightarrow D_{2}$ be a holomorphic mapping smooth up to the boundary, $f(0)=0$ and $V$ is an irreducible component of the branch locus $V_{f}$ such that $0 \in \bar{V}$. Then $V=D_{1} \cap\{(z, w) \in W: z=0\}$.

In what follows we denote by $\Gamma_{j}$ the boundary of $D_{j}$ near the origin. Recall that by the well-known argument [5] the set $E \subset \bar{V} \cap \Gamma_{1}$ of points where $V$ is a $C^{\infty}$ smooth manifold with boundary transversal to $\Gamma_{1}$ is open dense in $\bar{V} \cap \Gamma_{1}$ ([5] considers the strictly pseudoconvex case but the argument easily can be adapted for our case in view of the existence of holomorphic peak functions [9] and plurisubharmonic exhaustion functions [19], see also [6]).

First, we assume that $0 \in E$, i.e. $V$ is a $C^{\infty}$ smooth variety with boundary near the origin and transversal to $\Gamma_{1}$; the general case will be reduced to this one. We proceed the proof by contradiction. Assume that the statement is false. Since $V$ is an irreducible complex variety and a smooth manifold with boundary near 0 , there exists a sequence $\zeta^{\nu}=\left(a^{\nu}, b^{\nu}\right)$ in $\bar{V} \cap \Gamma_{1}$ converging to 0 and such that $a^{\nu} \neq 0$ for any $\nu$. Since $V$ is transversal to the boundary at 0 , it follows by the implicit function theorem that there exists a smoothly bounded domain $X$ in $\mathbb{C}, 0 \in \partial X$ and a neighborhood $U$ of the origin in $\mathbb{C}^{2}$ such that

$$
V \cap U=\{(z, w) \in U: z=h(w), w \in X\}
$$


where $h$ is a holomorphic function on $X$ smooth up to the boundary. We extend $h$ smoothly past the boundary and assume that it is defined in a neighborhood $Y$ of 0 .

Let $b^{\nu}=\alpha^{\nu}+i \beta^{\nu}$. Let us consider the translations $T^{\nu}:(z, w) \longrightarrow$ $\left(z, w+\alpha^{\nu}\right)$. Then the sequence $\left(T^{\nu}\right)$ converges uniformly to the identity on any compact subset of $\mathbb{C}^{2}$ and $\eta^{\nu}=T^{\nu^{-1}}\left(\zeta^{\nu}\right)=\left(a^{\nu}, i \beta^{\nu}\right)$. Set $f^{\nu}=$ $f \circ T^{\nu}$ and $W^{\nu}=T^{\nu^{-1}}(W)$. Then the sequence $f^{\nu}: D_{1} \cap W^{\nu} \longrightarrow D_{2}$ is a sequence of proper holomorphic mappings ; the sequence $\left(W^{\nu}\right)$ converges in the Hausdorff distance sense to $W$ and $\left(f^{\nu}\right)$ converges to $f$ uniformly on any compact subset of $\bar{D}_{1} \cap W$. We denote by $V^{\nu}$ the pullback $T^{\nu^{-1}}(V)$ which is contained in the branch locus of $f^{\nu}$. We have $\eta^{\nu} \in V^{\nu} \cap \Gamma_{1}$.

LEMMA 3.2. There exists a constant $C>0$ such that for any $\nu$ and any $(z, w) \in D_{1} \cap W^{\nu}$ one has

$$
C^{-1} \operatorname{dist}\left((z, w), \Gamma_{1}\right) \leq \operatorname{dist}\left(f^{\nu}((z, w)), \Gamma_{2}\right) \leq C \operatorname{dist}\left((z, w), \Gamma_{1}\right) .
$$

For the proof we observe that the statement holds for $f$ by the Hopf Lemma (see [2]) and the linear mappings $T^{\nu}$ preserve the distance to $\Gamma_{1}$ with a uniform constant independent of $\nu$.

We note that $V^{\nu} \cap W^{\nu}$ is defined by $\left\{(z, w) \in W^{\nu}: z=h^{\nu}(w)=\right.$ $\left.h\left(w-\alpha^{\nu}\right)\right\}$, where $h^{\nu}$ is holomorphic on $X^{\nu}=X-\alpha^{\nu}$ and smooth up to the boundary ; evidently, $h^{\nu}$ converges together with all derivatives uniformly to $h$ on any compact of $X$.

We set $\delta_{\nu}=\left|a^{\nu}\right|^{2 m}, p^{\nu}=\left(0,-\delta_{\nu} i\right)$. Considering the Taylor expansion of $h^{\nu}$ near $\eta^{\nu}$ we get $z-a^{\nu}=\lambda_{\nu}\left(w-i \beta^{\nu}\right)+\psi^{\nu}(w)$, where $\psi^{\nu}$ is smooth function in a fixed neighborhood of 0 and there exists a constant $M>0$ such that $\left|\psi^{\nu}(w)\right| \leq M\left|w-i \beta^{\nu}\right|^{2}$ for any $\nu$. Note also that the sequence $\left(\lambda_{\nu}\right)$ is bounded by $(1)$.

Fix $\alpha<0$. Let us slice $V^{\nu}$ by a complex line $w=i \alpha \delta_{\nu}+i \beta^{\nu}$. For $\nu$ large enough the intersection point is (by uniformity of neighborhoods) $\left(z^{\nu}, w^{\nu}\right)=\left(a^{\nu}+i \alpha \delta_{\nu} \lambda_{\nu}+o\left(\delta_{\nu}\right), i \alpha \delta_{\nu}+i \beta^{\nu}\right)$.

Set $r_{1}(z, w)=\operatorname{Im} w+P_{2 m}(z)+\varphi(z)$. Since $r_{1}\left(a^{\nu}, b^{\nu}\right)=0$, we have $r_{1}\left(z^{\nu}, w^{\nu}\right)=\delta_{\nu} \alpha+o\left(\delta_{\nu}\right)<0$ for $\nu$ large enough. Hence, $\left(z^{\nu}, w^{\nu}\right)$ is in $D_{1} \cap W^{\nu}$ for $\nu$ large enough.

Now we can apply a version of the scaling construction developed in $[17,18]$. We need the following well-known statement basic for analysis on pseudoconvex domains of finite type (see $[16,20]$ ). 
Let $\Omega$ be a domain in $\mathbb{C}^{2}$ with $C^{\infty}$ smooth boundary near a point $p \in \partial \Omega$ of finite type $2 k$. Then there exists a neighborhood $U$ of $p$ with the following properties:

(a) there exists a local biholomorphic change of coordinates such that in the new coordinates we have

$$
\Omega \cap U=\left\{(z, w) \in \mathbb{C}^{2} \mid r(z)=\operatorname{Im} w+\theta(z, \operatorname{Rew})<0\right\}
$$

where $\theta \in C^{\infty}$ and vanishes at the origin with the order of (at least) 2 ;

(b) there exists a mapping $\Phi: \mathbb{C}^{2} \times U \rightarrow \mathbb{C}^{2}$ of class $C^{\infty}$ such that

(b1) $\Phi(\bullet, \xi)$ is a polynomial and $\Phi(\xi, \xi)=0$;

(b2) there exists a neighborhood $V$ of $p$ and $V_{\xi} \ni \xi$ such that $V \subset$ $V_{\xi} \subset U, \Phi(\bullet, \xi)$ is a biholomorphism from $V_{\xi}$ onto the unit ball $\mathbb{B} \subset \mathbb{C}^{2}$; the mapping $(t, \xi) \mapsto \Phi(\bullet, \xi)^{-1}(0, i t)$ is a diffeomorphism between $(-1,1) \times$ $(\partial \Omega \cap V)$ and $V$ (this implies by continuity that there exists an open cone $C_{0}$ with vertex at the origin in the direction of $\operatorname{Im} w$ and an open cone $C_{\xi}$ with vertex on $\xi$ in the direction of the inward normal at $\xi$ and of the vertex angle independent of $\xi$ such that $\left.\Phi\left(\left(C_{\xi}\right), \xi\right) \subset C_{0}\right)$.

(b3) one has

$$
\begin{aligned}
& r \circ \Phi(\bullet, \xi)^{-1}-r(\xi)=\operatorname{Im} w+\sum_{\ell=2}^{2 k} P_{\ell}(z, \xi)+(\operatorname{Re} w) \sum_{\ell=1}^{k} Q_{\ell}(z, \xi) \\
& +\sigma_{2 k+1}(z, \xi)+\sigma_{2}(\operatorname{Re} w, \xi)+\left(\operatorname{Im} z_{2}\right) \sigma_{k+1}(z, \xi)
\end{aligned}
$$

on $V \times \mathbb{B}$; here $P_{\ell}$ and $Q_{\ell}$ are homogeneous polynomials in $z$ and $\bar{z}$ of degree $\ell$ without purely harmonic terms; $\sigma_{i}(v, \xi)$ vanishes of order $i$ in $v$;

(c) one has $\inf _{\xi} \sup _{\ell}\left\|P_{\ell}(\bullet, \xi)\right\|>0$, where \|\| is the norm of homogeneous polynomials.

For $\varepsilon>0$ we set $\tau(\xi, \varepsilon)=\min _{\ell=2, \cdots, 2 k}\left(\frac{\varepsilon}{\left\|P_{\ell}(\bullet, \xi)\right\|}\right)^{1 / \ell}$.

We suppose also that $\Gamma_{2}$ is of type $2 k$ near the origin.

Set $q^{\nu}=f^{\nu}\left(p^{\nu}\right)$. We denote by $\omega^{\nu}$ the point of $\Gamma_{2}$ closest to $q^{\nu}$; set also $\gamma_{\nu}=\operatorname{dist}\left(q^{\nu}, \Gamma_{2}\right)=\left|q^{\nu}-\omega^{\nu}\right|$. Let $g^{\nu}$ denote the polynomial biholomorphism $\Phi\left(\bullet, \omega^{\nu}\right)$ corresponding to $\Gamma_{2}$. Without loss of generality one can assume that $g^{\nu} \rightarrow$ id uniformly on compact subsets of $\mathbb{C}^{2}$ as $\nu \rightarrow \infty$.

Let us consider the holomorphic mappings $\tilde{f}^{\nu}=g^{\nu} \circ f: D_{1} \rightarrow g^{\nu}\left(D_{2}\right)$ and the following dilations of coordinates : $A^{\nu}:(z, w) \mapsto\left(\delta_{\nu}^{-1 / 2 m} z, \delta_{\nu}^{-1} w\right)$ and $B^{\nu}:(z, w) \mapsto\left(\tau\left(\omega^{\nu}, \gamma_{\nu}\right)^{-1} z, \gamma_{\nu}^{-1} w\right)$. We set $D_{1}^{\nu}=A^{\nu}\left(D_{1}\right), D_{2}^{\nu}=$ 
$B^{\nu} \circ g^{\nu}\left(D_{2}\right)$ and consider the mappings

$$
F^{\nu}=B^{\nu} \circ g^{\nu} \circ f^{\nu} \circ\left(A^{\nu}\right)^{-1}=B^{\nu} \circ \tilde{f}^{\nu} \circ\left(A^{\nu}\right)^{-1}: D_{1}^{\nu} \rightarrow D_{2}^{\nu}
$$

Let also $r_{1}^{\nu}(z, w)=\delta_{\nu}^{-1} r_{1} \circ\left(A^{\nu}\right)^{-1}=\delta_{\nu}^{-1} r_{1}\left(\delta_{\nu}^{1 / 2 m} z, \delta_{\nu} w\right)$ and $r_{2}^{\nu}=$ $\gamma_{\nu}^{-1} r_{2} \circ\left(g^{\nu}\right)^{-1} \circ\left(B^{\nu}\right)^{-1}=\gamma_{\nu}^{-1} r_{2}^{\nu}\left(\tau\left(\omega^{\nu}, \gamma_{\nu}\right) z, \gamma_{\nu} w\right)$.

Since $\Gamma_{2}$ is of type $2 k$ at the origin, one has

$$
r_{2}^{\nu}=\operatorname{Im} w+\gamma_{\nu}^{-1}\left(\sum_{\ell=2}^{2 k}\left(\tau\left(\omega^{\nu}, \gamma_{\nu}\right)\right)^{\ell} P_{2, \ell}\left(\omega^{\nu}, z\right)\right)+R^{\nu}
$$

where the sequence $\left(R^{\nu}\right)_{\nu}$ converges to zero uniformly on compact subsets of $\mathbb{C}^{2}$ as $\nu \rightarrow \infty$ (see $\left.[17,18]\right)$.

Passing to the subsequence, one can assume that the polynomials $\gamma_{\nu}^{-1} \Sigma_{\ell=2}^{2 k} P_{2, \ell}\left(\omega^{\nu}, \tau^{\ell}\left(\omega^{\nu}, \gamma_{\nu}\right) z\right)$ converge uniformly on compact subsets of $\mathbb{C}$ to a nonzero real polynomial $Q$ of degree $\leq 2 k$ that contains no purely harmonic terms. Let us consider the domain $\Omega_{2}=\left\{w \in \mathbb{C}^{2} \mid \psi(z, w)=\right.$ $\operatorname{Im} w+Q(z)<0\}$.

The sequence $\left(r_{2}^{\nu}\right)$ converges to the function $\psi$ uniformly together with all derivatives of any order ; hence $\Omega_{2}$ is pseudoconvex as a smooth limit of pseudoconvex domains. In particular, $Q$ is a subharmonic polynomial on $\mathbb{C}$.

Similarly, we have that the sequence $\left(r_{1}^{\nu}\right)$ converges uniformly on compact subsets of $\mathbb{C}^{2}$ to the function $\phi=\operatorname{Im} w+P_{2 m}(z)$ (in what follows we write simply $P$ ). It is worth to note that $P$ is a homogeneous polynomial. We define the domain $\Omega_{1}=\left\{(z, w) \in \mathbb{C}^{2} \mid \phi(z)<0\right\}$.

Now quite similarly to [17], it follows by [12] that there exists a subsequence of $\left(F^{\nu}\right)_{\nu}$ uniformly converging on compact subsets of $\Omega_{1}$. Thus, without loss of generality one can assume that $\left(F^{\nu}\right)_{\nu}$ converges uniformly on compact subsets of $\Omega_{1}$ to a holomorphic mapping $F$. This was shown in $[17,18]$ that $F$ takes its values in $\Omega_{2}$ and, moreover, one has $\psi(F(z, w)) \leq$ $C(R) \phi(z, w)$ for any $R>0$ and $z \in \Omega_{1} \cap R \mathbb{B}$ (here and below $\mathbb{B}$ denotes the unit ball of $\left.\mathbb{C}^{2}\right)$.

We have $A^{\nu}\left(p^{\nu}\right)=(0,-i), A^{\nu}\left(\eta^{\nu}\right)=\left(e^{i \theta_{\nu}}, i \beta^{\nu} \delta_{\nu}^{-1}\right)$. But $\beta^{\nu}=P_{2 m}\left(a^{\nu}\right)$ $+o\left(\left|a^{\nu}\right|^{2 m}\right)$ and by the choice of $\delta_{\nu}$ the sequence $\beta^{\nu} \delta_{\nu}^{-1}$ tends to a finite point $\tau$. Therefore,

$$
A^{\nu}\left(z^{\nu}, w^{\nu}\right)=\left(e^{i \theta_{\nu}}+i \alpha \delta_{\nu}^{1-(1 / 2 m)}+o\left(\delta_{\nu}^{1-(1 / 2 m)}\right), i \alpha+i \beta^{\nu} / \delta_{\nu}\right)
$$


This sequence tends to the point $q=\left(e^{i \theta}, i \alpha+i \tau\right)$. Since $\left(e^{i \theta}, i \tau\right)$ is in the boundary of the limit model domain $\Omega_{1}$, the point $q$ is in $\Omega_{1}$.

Since the limit mapping $F: \Omega_{1} \longrightarrow \Omega_{2}$ satisfies conditions of Proposition 2.1 and $P_{2 m}$ is a homogeneous polynomial, by the previous section we obtain that $V_{F}=\{z=0\}$. But by the construction $q$ is in $V_{F}$ : a contradiction. This proves the proposition 3.1 in the case where $V$ is a smooth variety with boundary.

Suppose now that 0 is a point of $\left(\bar{V} \cap \Gamma_{1}\right) \backslash E$. Then we take a regular point $(a, b) \in E$ of type $2 s$ and consider the polynomial change of variables $T:\left(z^{\prime}, w^{\prime}\right) \longrightarrow(z, w)=\left(z^{\prime}+a, w^{\prime}+b+Q\left(z^{\prime}\right)\right)$, where the polynomial $Q$ is chosen such that $r_{1} \circ T=\operatorname{Im} w^{\prime}+R_{2 s}\left(z^{\prime}\right)+\varphi^{\prime}\left(z^{\prime}\right)$, where $R_{2 s}$ is a homogeneous subharmonic polynomial of degree $2 s$ without purely harmonic terms and $\varphi^{\prime}\left(z^{\prime}\right)=o\left(\left|z^{\prime}\right|^{2 s}\right)$. Then 0 is the regular point for the branch locus of the mapping $f \circ T$ and as it was just shown $J_{f \circ T}=\left\{z^{\prime}=0\right\}$ near the origin. This implies that (in the old coordinates) $V$ coincides with $\{(z, w): z=a\}$ near $(a, b)$. Since $V$ is irreducible, $V$ coincides with this line everywhere in $U$, and hence necessarily $a=0$. This completes the proof of Proposition 3.1 .

Q.E.D.

We can prove now our first main result.

Proof of Theorem 1.1.

By [4], $f$ is smooth up to the boundary. Let $V$ be an irreducible component of $V_{f}$ and $p \in \partial V$ be a boundary point of $V$. It follows by [27] that there exists a neighborhood $W$ of $p$ in $\mathbb{C}^{2}$, a neighborhood $U$ of 0 and a biholomorphic mapping $H: \Omega \cap W \longrightarrow \Omega^{\prime} \cap U$ smooth up to the boundary $\partial \Omega, H(p)=0$ such that $\Omega^{\prime} \cap U$ is rigid; $\mathbf{T}$ acts on $\Omega^{\prime} \cap U$ by translations $(z, w) \mapsto(z, w+t), t \in \mathbb{R}$. In view of [2] we can assume that the mapping $f \circ H^{-1}: \Omega^{\prime} \cap U \longrightarrow f(\Omega \cap V)$ is proper. Then it follows by Proposition 3.1 that $H(V \cap W)=\{(z, w) \in U: z=0\}$. But then $V \cap W=H^{-1}(\{z=0\})$ is a smooth manifold with boundary near $p$. This proves part (i) of Theorem 1.1 .

Since the circle $\mathbf{T}$ acts (locally) by translation on $\Omega^{\prime}$, we get that $(\partial V) \cap$ $W$ coincides with the orbit $\mathbf{T}(p) \cap W$. By compactness of $\partial V$ there exists a finite number of neighborhoods $W\left(p_{j}\right), j=1, \ldots, N, p_{j} \in \partial V$ such that $\partial V \subset \cup_{1}^{N} W\left(p_{j}\right)$ and for every $j$ the intersection $(\partial V) \cap W\left(p_{j}\right)$ is equal to the orbit $\mathbf{T}\left(p_{j}\right) \cap W\left(p_{j}\right)$. Hence, $\partial V$ is contained in a finite union of disjoint orbits. 
In order to show the inverse inclusion, we note that for any point $p$ in $\partial \Omega$ its orbit $\mathbf{T}(p)$ is a smooth connected compact curve; since after an one-sided biholomorphic change of coordinates the $\mathbf{T}$ action is given by translations and therefore this curve can be transformed to a real line, we get that for any point $a$ in $\mathbf{T}(p)$ there exists a neighborhood $U$ such that $\mathbf{T}(p) \cap U$ is the boundary of a complex 1-dimensional manifold in $\Omega \cap U$. Since $\mathbf{T}(p)$ is compact, there exists a neighborhood $W$ of $\mathbf{T}(p)$ such that $\mathbf{T}(p)$ is the boundary of a (connected) complex 1-dimensional manifold in $\Omega \cap W$ denoted by $\mathbf{T}(p)^{\mathbb{C}}$. If the intersection $\bar{V} \cap \mathbf{T}(p)$ contains a point $a$, then there exists a neighborhood $U$ of $a$ such that $V \cap U$ coincides with $\mathbf{T}(p)^{\mathbb{C}}$ on $U$. Since $\mathbf{T}(p)^{\mathbb{C}}$ is irreducible, it is contained in $V$ by the uniqueness theorem. Hence, $\mathbf{T}(p)$ is contained in $\bar{V}$. This completes the proof of part (ii).

\section{$\S 4$. Mappings from circular domains}

Important special cases of domains with $\mathbf{T}$-action arise when the action is linear; classical examples are provided by circular domains. This section is devoted to the proof of our second main result Theorem 1.2. In what follows by a disc in $\mathbb{C}^{2}$ we mean a linear disc, i.e. the image of the unit disc in $\mathbb{C}$ under a linear mapping from $\mathbb{C}$ to $\mathbb{C}^{2}$; in particular, such a disc contains the origin.

LEMMA 4.1. Let $f: \Omega \longrightarrow D$ be a proper holomorphic mapping between two smoothly bounded pseudoconvex finite type domains in $\mathbb{C}^{2}$. Suppose that $\Omega$ is a complete circular domain. Then the branch locus $V_{f}$ is a finite union of discs.

Proof. Since $\Omega$ is pseudoconvex, for every point $p \in \partial \Omega$ there exists a neighborhood $U$ and a defining function $\rho$ such that $-(-\rho)^{\alpha}$ is a plurisubharmonic function on $\Omega \cap U$ (shrinking $U$ if necessarily, one can take $\alpha \in(0,1)$ arbitrarily close to 1$)$ [19]. Since $\Omega$ is a complete circular domain, every $\mathbf{T}$-orbit is a circle in the boundary which bounds a complex disc in $\Omega$; it follows by the Hopf lemma that this disc is transversal to the boundary and hence the $\mathbf{T}$-action is transversal. Therefore, Theorem 1.1 implies that $\overline{V_{f}} \cap \partial \Omega$ is a finite union of circles. Then $V_{f}$ coincides with the union of corresponding discs (say, by the maximum principle).

Q.E.D. 
In the case of proper self-mappings the last proposition gives surprisingly strong corollaries which allow to prove our second main result.

Proof of Theorem 1.2.

Suppose that the branch locus $V_{f}$ is not empty. The first step of the proof of Theorem 1.2 is the following

LEMMA 4.2. The mapping $f$ is polynomial homogeneous.

Proof. First, we show that $f$ is a polynomial mapping. We denote by $f^{(n)}$ the $n$-th iteration of $f$. It follows by Lemma 4.1 that for every $n$ the branch locus $V_{f^{(n)}}$ is a finite union of discs .

We claim that there exists a sequence $\left(L_{n}\right)$ of discs such that $L_{n} \subset$ $V_{f(n)}, L_{n+1} \subset f^{-1}\left(L_{n}\right)$.

We will construct the family $\left(L_{n}\right)$ by induction. For every $n$ we have $V_{f^{(n+1)}}=V_{f} \cup f^{-1}\left(V_{f(n)}\right)$. Fix any disc $L_{1}$ in $V_{f}$. Then $f^{-1}\left(L_{1}\right)$ is contained in $V_{f(2)}$ and contains the disc $L_{2}$. Assume that the discs $L_{1}, \ldots, L_{n}$ are defined. Then $f^{-1}\left(L_{n}\right)$ is contained in $f^{-1}\left(V_{\left.f^{(} n\right)}\right) \subset V_{f^{n+1}}$. So there exists a disc $L_{n+1}$ such that $L_{n+1} \subset f^{-1}\left(L_{n}\right)$. Note that since every restriction $f: L_{n} \longrightarrow L_{n-1}$ is proper and $f\left(L_{n}\right) \subset L_{n-1}$, we have $f\left(L_{n}\right)=L_{n-1}$. We note that the discs $\left(L_{n}\right)$ are distinct. Indeed, suppose by contradiction that $m$ is the first integer such that there exists $p$ with $L_{m}=L_{m+p}$. If $m \geq 2$, we have $f\left(L_{m}\right)=f\left(L_{m+p}\right)$ and so $L_{m-1}=L_{m+p-1}$. This contradicts the definition of $m$. So $m=1$.

Let $\tau_{\partial \Omega}(p)$ be the order of vanishing of the Levi determinant introduced in section 2. Since $\tau$ is invariant with respect to the $\mathbf{T}$-action, $\tau$ is constant on every $\partial L_{n}$. We denote it by $\tau_{n}$. Since $L_{n+1}$ is contained in $f^{-1}\left(L_{n}\right)$, the sequence $\left(\tau_{n}\right)_{n}$ is increasing. The domain $\Omega$ is of finite type, therefore the sequence $\left(\tau_{n}\right)$ is bounded, so it is a constant for $n$ sufficiently large. Let $n_{0}$ be the first integer such that $\tau_{n}=\tau_{n_{0}}$ if $n \geq n_{0}$. Given $n \geq n_{0}$, for $z \in \partial L_{n+1}$ we have $\tau_{n+1}=\tau_{\partial \Omega}(z) \geq \tau_{\partial \Omega}(f(z))=\tau_{n}=\tau_{n+1}$. Hence $f$ is locally biholomorphic at $z$ and $z$ is not in $V_{f}$; but then $z$ cannot be in $L_{1}$. Thus $L_{2} \neq L_{n}$ for any $n$ large enough. This is a contradiction. So the discs $L_{n}$ are different. In particular, since $f\left(L_{n+1}\right)=L_{n}$ is proper for every $n$ we obtain that $f(0)=0$. There exists a positive integer $n_{0}$ such that for every $n \geq n_{0}$ the sequence $\left(\tau_{n}\right)_{n}$ is constant and $L_{n} \cap V_{f}=\{0\}$ (since $V_{f}$ is a finite union of discs). Consider a sequence of points $\left(p_{n}\right)_{n}$ in $\partial \Omega$ such that $p_{n}$ is in $L_{n}$ for every $n$. The restriction $f: L_{n+1} \rightarrow L_{n}$ is proper, $f(0)=0$ and, since $f$ is locally biholomorphic at $L_{n+1} \backslash\{0\}$, the 
derivative of the restriction $f_{\left.\right|_{L_{n+1}}}$ cannot vanish at a point different of the origin. Then we have $f\left(\lambda p_{n+1}\right)=c_{n} \lambda^{k_{n}} p_{n}$ where $\lambda \in \mathbb{C}:|\lambda|<1, c_{n}$ is a constant, an integer $k_{n}$ is smaller than the order of vanishing or $(f)$ of $f$ at the origin. Fix $r>0$ such that the ball $r \mathbb{B}$ is contained in $\Omega$ and consider the decomposition of $f$ in homogeneous polynomials $f=\Sigma f_{k}$ on $r \mathbb{B}$ with $f_{k}(\lambda z)=\lambda^{k} f_{k}(z)$ for any $z$. Since $f\left(\lambda p_{n+1}\right)=c_{n} \lambda^{k_{n}} p_{n}$, we have $\sum \lambda^{k} f_{k}\left(p_{n+1}\right)=c_{n} \lambda^{k_{n}} p_{n}$ for every $\lambda$ in a neighborhood of the origin in $\mathbb{C}$ independent of $n$; therefore $f_{k}\left(p_{n+1}\right)=0$ for $k \geq \operatorname{or}(f)+1$ for any $n \geq n_{0}$. Thus, $f_{k}$ vanishes on every $L_{n}, n \geq n_{0}$ for $k \geq$ or $(f)+1$. Since the lines $L_{n}$ are different, every homogeneous polynomial $f_{k}$ is zero. Thus, $f$ is a polynomial of degree $\leq$ or $(f)$.

Finally, let us show that $f$ is homogeneous. For $k \leq$ or $(f)$, let $N_{k}$ denote the set of positive integer $n$ such that $f\left(\lambda p_{n+1}\right)=c_{n} \lambda^{k} p_{n}$. There exists $k_{0}$ such that $N_{k_{0}}$ is infinite. For every $j$ different from $k_{0}$ we have $f_{j}\left(\lambda p_{n+1}\right)=0$ for any $n \in \mathbb{N}_{k_{0}}$ and since $N_{k_{0}}$ is infinite, we obtain that $f_{j}=0$; hence $f=f_{k_{0}}$ and $f$ is homogeneous.

Q.E.D.

The second basic step in our proof of Theorem 1.2 is an application of complex dynamics arguments. We refer the reader to $[15,23,21]$ for standard definitions.

Since $f: \Omega \longrightarrow \Omega$ is proper and homogeneous, it follows that $f$ is nondegenerate, i.e. $f^{-1}(0)=0$. Let $\Omega_{f}$ denote the basin of attraction for $f$. Note that this is a complete circular domain (see also [23]).

LEMMA 4.3. One has $\Omega=\Omega_{f}$.

Proof. One can assume that $\left(f^{(k)}\right)$ converges to $F$ on $\Omega$. For every $\lambda \in \mathbb{C},|\lambda|<1$ one has $f^{(k)}(\lambda z) \longrightarrow F(\lambda z)$ as $k \longrightarrow \infty$. But $f^{(k)}$ is homogeneous of degree $d^{k}$ and $f^{(k)}(\lambda z)=\lambda^{d^{k}} f^{(k)}(z)$ which converges to 0 . Hence, $F=0$ on $\Omega$ and $\Omega \subset \Omega_{f}$. But $f$ is proper and $f(\partial \Omega) \subset \partial \Omega$. Hence, $\Omega_{f}$ is contained in $\Omega$.

Q.E.D.

In order to prove Theorem 1.2 , it suffices to prove that $V_{f}$ is empty $([26])$. Suppose by contradiction that it is not so. Then as it was just shown, $\mathrm{f}$ is a homogeneous polynomial, which is not linear.

We denote by $\pi: \mathbb{C}^{2} \backslash\{0\} \longrightarrow \mathbb{C} P$ the canonical projection. Since $f$ is nondegenerate, it takes lines to lines in $\mathbb{C}^{2}$ and naturally induces a rational 
mapping $\varphi: \mathbb{C} P \longrightarrow \mathbb{C} P$ on the projective space. We claim that its Julia set $J_{\varphi}$ does not coincide with $\mathbb{C} P$. For the proof we apply an argument of [14]. Suppose by contradiction it does. This is known (see [15], pp.56-58) that in this case for every point $a \in J_{\varphi}$ there exists a neighborhood $U$ and a positive integer $n$ such that $\cup_{k=1}^{n} \varphi^{(k)}(U)$ covers $\mathbb{C} P$. Take $a$ such that $\pi^{-1}(a)$ contains a strictly pseudoconvex point $p$ in $\partial \Omega$. Then there exists a neighborhood $W$ of $p$ in $\mathbb{C}^{2}$ such that $\cup_{k=1}^{n} f^{(k)}(W)$ covers $\partial \Omega$. Since $f$ takes any strictly pseudoconvex point to a strictly pseudoconvex one, we get that $\Omega$ is strictly pseudoconvex and by [26] $V_{f}$ is empty: a contradiction.

Thus, $J_{\varphi}$ is different from $\mathbb{C} P$. But then by the classical results $J_{\varphi}$ is a closed subset of $\mathbb{C} P$ with empty interior ([15], Theorem 1.9). Therefore $\partial \Omega \backslash \pi^{-1}\left(J_{\varphi}\right)$ is a nonempty open subset of $\partial \Omega$ which in view of [23], Proposition 7.1, is foliated by Riemann surfaces: this is impossible since $\Omega$ is of finite type. This completes the proof of the theorem.

In conclusion we note that if $\Omega$ is a circular, but not complete circular domain, then the circled action in general is not transversal as shows the domain $D=\left(|z|^{2}-1\right)^{2}+\left(|w|^{2}-1\right)^{2}<\varepsilon$ for $\varepsilon>0$ small enough. But if the action is transversal, the former proof is still valid with slight modifications (one has consider proper holomorphic mappings of annuli in linear sections).

\section{REFERENCES}

[1] H. Alexander, Proper holomorphic mappings in $\mathbb{C}^{n}$, Indiana Univ. Math. J., 26 (1977), 137-146.

[2] M. Baouendi, S. Bell and L.P. Rothschild, Mappings of three-dimensional CR manifolds and their holomorphic extension, Duke Math. J., 56 (1988), 503-530.

[3] D. Barrett, Regularity of the Bergman projection on domains with transverse symmetries, Math. Ann., 258 (1982), 441-446.

[4] - Boundary analyticity of proper holomorphic maps of domains with nonanalytic boundaries, Math. Ann., 263 (1983), 474-482.

[5] E. Bedford, Proper holomorphic mappings from strongly pseudoconvex domains, Duke Math. J., 49 (1982), 477-484.

[6] - Proper holomorphic mappings from domains with real analytic boundary, Amer. J. Math., 106 (1984), 745-760.

[7] Action of the automorphisms of a smooth domain in $\mathbb{C}^{n}$, Proc. Amer. Math. Soc., 93 (1985), 365-400.

[8] E. Bedford and S. Bell, Proper self-maps of weakly pseudoconvex domains, Math. Ann., 261 (1982), 505-518.

[9] E. Bedford and J.E. Fornaess, A construction of peak functions on weakly pseudoconvex domains, Ann. Math., 270 (1978), 555-568. 
[10] S. Bell, Local boundary behavior of proper holomorphic mappings, Proc. Symp. Pure Math., 41 (1984), 1-7.

[11] S. Bell and D. Catlin, Regularity of CR mappings, Math. Z., 199 (1988), 357-368..

[12] F. Berteloot and J. Coeuré, Domaines de $\mathbb{C}^{2}$ pseudoconvexes et de type fini ayant un groupe non compact d'automorphismes, Ann. Inst. Fourier, 41 (1991), 77-86.

[13] F. Berteloot and S. Pinchuk, Proper holomorphic mappings between bounded complete reinhardt domains in $\mathbb{C}^{2}$, Math. Z., 219 (1995), 343-356.

[14] F. Beteloot and J.J. Loeb, A geometrical characterization of Lattes rational map, Preprint. Univ. d'Angers, 1996.

[15] L. Carleson and T. Gamelin, Complex dynamics, Springer-Verlag, 1993.

[16] D. Catlin, Estimates on invariant metrics on pseudoconvex domains of dimension two, Math. Z., 200 (1989), 429-466.

[17] B. Coupet, S. Pinchuk and A.Sukhov, On boundary rigidity and regularity of holomorphic mappings, Internat. J. Math., 7 (1996), 617-643.

[18] B. Coupet and A. Sukhov, On CR mappings between pseudoconvex hypersurfaces of finite type in $\mathbb{C}^{2}$, Duke Math. J., 88 (1997), 281-304.

[19] K. Diederich and J.E. Fornaess, Pseudoconvex domains: Bounded strictly plurisubharmonic exhaustion functions, Invent. math., 39 (1977), 371-384.

[20] J.E. Fornaess and N.Sibony, Construction of P.S.H. functions on weakly pseudoconvex domains, Duke Math. J., 58 (1989), 633-656.

[21] same, Complex dynamics in higher dimension I, Asterisque, 221 (1994), 201-231.

[22] X. Huang and Y. Pan, Proper holomorphic mappings between real analytic domains in $\mathbb{C}^{n}$, Duke Math. J., 82 (1996), 437-446.

[23] J. Hubbard and P. Papadopol, Superattractive fixed points in $\mathbb{C}^{n}$, Indiana Univ. Math. J., 43 (1994), 321-365.

[24] D. Mumford, Alebraic geometry I. Complex projective varieties, Springer Verlag, 1976.

[25] Y. Pan, Proper holomorphic self-mappings of Reinhardt domains, Math. Z., 208 (1991), 289-295.

[26] S. Pinchuk, Holomorphic inequivalence of some classes of domains in $\mathbb{C}^{n}$, Math. USSR Sb., 39 (1981), 61-86.

[27] N. Tanaka, On the pseudo-conformal geometry of hypersurfaces of the space of $n$ complex variables, J. Math. Soc. Japan, 14 (1962), 397-429. 
Bernard Coupet

LATP, CNRS/ UMR n $n^{\circ} 6632$

CMI, Université de Provence

39, rue Joliot Curie

13453 Marseille cedex 13

France

Yifei Pan

Department of Mathematics

Indiana University-

Purdue University Ft. Wayne

Ft. Wayne, IN 46805

U.S.A.

Alexandre Sukhov

LATP, CNRS/UMR n 6632

CMI, Université de Provence

39, rue Joliot Curie

13453 Marseille cedex 13

France 